\title{
3D Reconstruction from Hyperspectral Images
}

\author{
Ali Zia ${ }^{1} \quad$ Jie Liang $^{2} \quad$ Jun Zhou $^{1} \quad$ Yongsheng Gao $^{3}$ \\ ${ }^{1}$ School of Information and Communication Technology, Griffith University, Australia \\ ${ }^{2}$ Research School of Engineering, College of Engineering and Computer Science \\ Australian National University, Australia \\ ${ }^{3}$ School of Engineering, Griffith University, Australia \\ ali.zia@griffithuni.edu.au, u5153489@anu.edu.au, \{jun.zhou,yongsheng.gao\}@griffith.edu.au
}

\begin{abstract}
$3 D$ reconstruction from hyperspectral images has seldom been addressed in the literature. This is a challenging problem because $3 D$ models reconstructed from different spectral bands demonstrate different properties. If we use a single band or covert the hyperspectral image to grayscale image for the reconstruction, fine structural information may be lost. In this paper, we present a novel method to reconstruct a 3D model from hyperspectral images. Our proposed method first generates $3 D$ point sets from images at each wavelength using the typical structure from motion approach. A structural descriptor is developed to characterize the spatial relationship between the points, which allows robust point matching between two $3 D$ models at different wavelength. Then a $3 D$ registration method is introduced to combine all band-level models into a single and complete hyperspectral 3D model. As far as we know, this is the first attempt in reconstructing a complete $3 D$ model from hyperspectral images. This work allows fine structural-spectral information of an object be captured and integrated into the $3 D$ model, which can be used to support further research and applications.
\end{abstract}

\section{Introduction}

Hyperspectral images have been widely used in remote sensing, mining, and surveillance. It contains tens or hundreds continuous light wavelength indexed spectral bands, which provides abundant information on the spatial spectral distribution of object materials that is related to their physical, chemical, and geometrical property. Compared to RGB images, hyperspectral data has also shown great potential in 3D computer vision, particularly for the applications that require fine analysis of the spectral responses of object. In plant phenomics [6], spectral data of the plant surface can be used to analyze the biomass, or determine the location of sick leaves on the 3D model. In computer graphics, capturing spectral data on 3D objects have enabled producing images of high visual quality or mimicking the vision system of animals [12]. Examples can be further extended to culture heritage [11] and biomedical imaging [19].

Despite its full potential in computer vision, research on $3 \mathrm{D}$ reconstruction from hyperspectral imaging is still very limited. In most work that have incorporated hyperspectral data into 3D models, the 3D shapes were generated by range camera rather than from hyperspectral images directly. The spectral information were then mapped to the 3D shape. In the historical building reconstruction, multispectral data is combined with depth information captured by a range camera [3]. Kim et al. introduced a system for capturing spectral data on 3D objects [12]. A hyperspectral imager was used to acquire high spatial resolution band images from near-ultraviolet to near-infrared range. This imager was then integrated into a 3D scanning system to capture the spectral reflectance and fluorescence of objects. Similarly, Nieto et al. developed a hyperspectral 3D modelling system for the mining industry [18]. 3D model was generated based on depth data captured by a laser scanner, with hyperspectral image mapped to this 3D Model. Manabe et al presented an interesting approach to represent spectral information as 3D model. They first constructed 3D model using two different hardware and then mapped the spectral information to the 3D model [16]. This method does not explore relationship between spectra and structure. Different from all these methods, Liang et al proposed a method to build multiple 3D models of plant, each from a different band of a set of hyperspectral images captured at different viewing angles [13]. The results show that the 3D models reconstructed from different bands have demonstrated different properties which are complement to each other.

The differences between band-wise 3D models are caused by various reasons. The first reason is the that object surface has different reflectance property due to the variation of composition materials. Some information that is vis- 


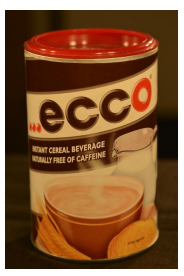

(a)

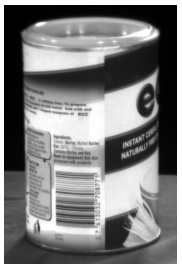

(e)

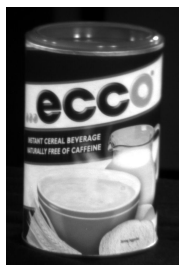

(b)

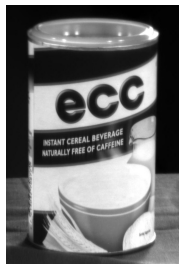

(f)

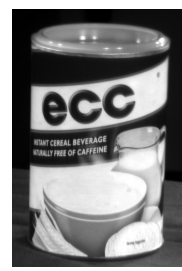

(c)

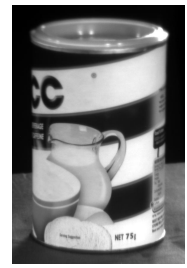

(g)

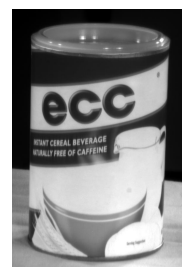

(d)

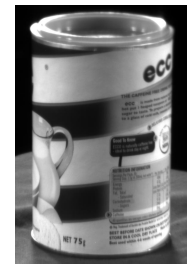

(h)

Figure 1. Examples of RGB and hyperspectral images. (a) RGB image. Band images captured at (b) 600nm, (c) 700nm, (d) 800nm from the same camera position, respectively; the second row shows band images captured at different camera positions, respectively.

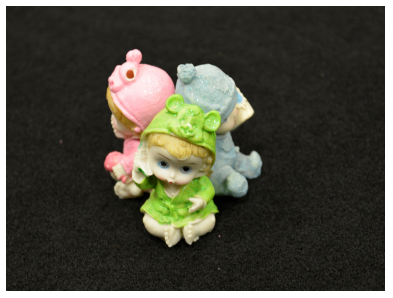

(a)

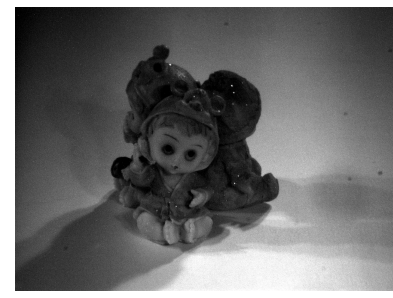

(b)

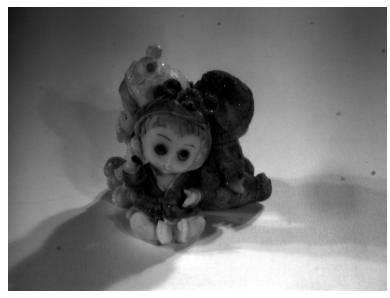

(c)

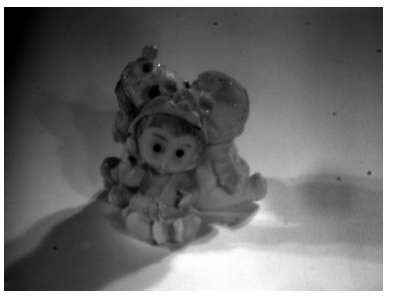

(d)

Figure 2. Examples of hyperspectral images with different focus and spectral features. (a) RGB image. Band images captured at (b) 580nm, (c) $700 \mathrm{~nm}$, (d) $860 \mathrm{~nm}$ from the same camera position, respectively.

ible in one band may become invisible in another band. An example on different band images of a coffee jar is shown in Fig. 1. The letter ' $O$ ' in the brand name is clearly visible in some bands whereas absent in others. The second reason is that point of focus changes due to change in wavelength [9]. This happens due to the fact that focal length of a lens is related to its refractive index which decreases with the increase of wavelength. Therefore, most available hyperspectral cameras can only be well focused on several bands and become out of focus in other bands. This can be observed in Fig. 2. Due to these factors, different feature points can be extracted from each band image, which produces different band-wise 3D models with inconsistent scales and point sets. These models cannot be merged directly to form a complete $3 \mathrm{D}$ model. One way to produce a single 3D model from hyperspectral images is merging all key points captured from different bands for 3D reconstruction. However, the fine structural and spectral information embedded in the band images are lost.

This paper aims to solve this problem and combine all band-level 3D models into a complete hyperspectral 3D model. Our method first generates wavelength indexed 3D point sets from a sequence of hyperspectral images. Then a structural descriptor is developed to characterize the spatial relationship of points within the same set, which can then be used to match points between two 3D models generated at different bands. This allows 3D models be registered and form the complete hyperspectral 3D model.

As far as we know, our work is the very first attempt in generating a complete 3D model from hyperspectral images. It not only generates band-wise 3D models, but also allows fine structural spectral information of an object to be captured and integrated into the fully reconstructed 3D model. This is beyond the capability of the traditional 3D reconstruction from RGB images. This paper also proposes a 3D registration algorithm which is accurate and efficient in merging band-wise models, which facilitates the analysis on how structural information are contributed by different band models.

The rest of the paper is organized as follows. Section 2 introduces how hyperspectral data was collected. Section 3 describe the 3D reconstruction method from hyperspectral images. Experimental results on both simulated data and real hyperspectral data are given and analyzed in Section 4, followed by conclusions in Section 5. 


\section{Hyperspectral data}

We have generated image sequences using a hyperspectral imaging system that consists of an acousto-optical tunable filter (AOTF) and a high sensitive visible to infrared camera. The filter supports imaging from $400 \mathrm{~nm}$ to $1000 \mathrm{~nm}$ at $20 \mathrm{~nm}$ in spectral resolution with a control unit tuning the wavelength of light that is allowed to reach the camera. By scanning through the visible to infrared wavelength, grayscale images can be generated to form different bands of the hyperspectral image. The output of the imaging process is a data cube with the first two dimensions showing the spatial positions of pixels, and the third dimension indexing the bands. Therefore, each pixel on the image is a vector of responses across the visible to infrared spectrum.

To capture the hyperspectral image sequence of objects, we have positioned the hyperspectral camera at same height from different view points relative to the object. These positions are around the object and the distance between the camera and the object varies slightly. At each position, we also took an RGB image. In total, we collected 30 hyperspectral images and 30 RGB images, which were used for image based 3D reconstruction. We have captured image sequences of 5 objects, including coffee can, ceramic dolls , toy monkey, plant, and toy fish. Due to the space limit, we only show the sample images of the coffee can and ceramic dolls in Fig. 1 and Fig. 2 respectively.

\section{3D Reconstruction from Hyperspectral Im- ages}

Our 3D reconstruction method starts from building separate 3D models from each hyperspectral band. This can be implemented by the standard image-based 3D modelling approach [7]. Let the band-wise 3D model be $\mathbf{X}(\lambda), \lambda=$ $1, \ldots, M$ be the wavelength indexed bands, then the goal is to build a complete hyperspectral 3D model

$$
\mathcal{Z}=\sum_{\lambda} \mathcal{X}(\lambda)
$$

where $\mathcal{X}(\lambda)$ is the consistently registered band-wise 3D model. Because hyperspectral band images captured at different wavelength ranges may differ significantly, it is very likely that not enough common points can be generated for the correspondence detection. A natural solution is to adopt a progressive strategy so that each $3 \mathrm{D}$ model is only matched to a model with the highest number of common points. Nonetheless, grouping and matching of bands shall be performed in an optimized order. Detailed discussion on the merging strategy will be given in Section 3.3.

In the following, we give detailed description on the proposed 3D registration and model fusion method.

\subsection{A Descriptor for Point-wise Correspondence}

Given two sets of 3D points extracted from different bands, the first step to match the 3D models is finding the point-wise correspondences. One of the widely used method is Iterative Closest Point (ICP) [2], which registers two point sets by minimizing the distances between the points in the first set to the corresponding points or the surface of the second set. The problem of ICP is that it is often suffered from scale differences between models. To solve this problem, various scale estimation methods have been proposed [21, 14], these methods require a rough prealignment of the point sets, which is traditionally based on point-wise Euclidean distance.

In this paper, we present a point descriptor that characterizes the statistical information on the structure of the 3D model, i.e., distribution of spatial relationship between a point and all the rest points in the set. A frequency histogram is generated as the descriptor, which allows fast point matching for point-wise correspondences. Mathematically, this idea is close to the structural criterion as proposed in [1], however, not constrained by the 2D shapes. It should be noted that histogram representations for 3D point have been reported in the literature but are all based on surface norm $[15,20]$. Our method, however, directly operate on the Euclidean distance between points.

Given a set of 3D data points $\mathbf{X}=\left\{\mathbf{x}_{1}, \mathbf{x}_{2}, \ldots, \mathbf{x}_{m}\right\}$. Let the Euclidean distance between $\mathbf{x}_{i}$ and $\mathbf{x}_{j}$ be $d_{i, j}$. A point histogram $H_{\mathbf{x}_{i}}$ of $\mathbf{x}_{i}$ is a histogram over the distribution of $d_{i, j}$ for all $j \neq i$. Let this set of distances be $D$. A straightforward way of constructing the point histogram is to find the maximum value of all distances $d_{i, j}^{*}$, and then divide $d_{i, j}^{*}$ into $n$ bins with equal distance intervals. Each entry in $D$ can then be assigned to the histogram bin corresponding to the distance interval it belongs to. Therefore,

$$
H_{\mathbf{x}_{i}(k)}=\#\left\{j \neq i: d_{i, j} \in \operatorname{bin}(k)\right\}
$$

where $\#\{$.$\} counts the number of elements. This generates$ point histograms each of which is comprised by the same number of bins. Note that $H_{\mathbf{x}_{i}}$ is invariant to the scale of the 3D model because each entry of the histogram describes the statistics on the number of points with certain relative distances to $x_{i}$.

A problem of this representation is when the number of points in two point sets are different, the height of the histogram will be different. To solve this problem, we normalized the histogram by the sum of all entries. Then the normalized point histogram becomes $\tilde{H}_{\mathbf{x}_{i}}$, whose $k^{t h}$ entry is

$$
\tilde{H}_{\mathbf{x}_{i}}(k)=\frac{H_{\mathbf{x}_{i}}(k)}{\sum_{k} H_{\mathbf{x}_{i}}(k)}
$$

where $H_{\mathbf{x}_{i}}(k)$ is the $k^{t h}$ entry of $H_{\mathbf{x}_{i}}$. In this way, we can use the point histogram to represent the structural information about $\mathbf{x}_{i}$, and the whole 3D point set. 
Based on the above point descriptor, given a point $\mathbf{x}_{i}$ in the first point set $\mathbf{X}$, and a point $\mathbf{y}_{j}$ in the second point set $\mathbf{Y}$. Let $C\left(\mathbf{x}_{i}, \mathbf{y}_{j}\right)$ be the similarity between two points, then correlation coefficient can be used for the calculation

$$
\begin{aligned}
C\left(\mathbf{x}_{i}, \mathbf{y}_{j}\right) & =\frac{\operatorname{cov}\left(\tilde{H}_{\mathbf{x}_{i}}, \tilde{H}_{\mathbf{y}_{i}}\right)}{\sigma_{\tilde{H}_{\mathbf{x}_{i}}} \sigma_{\tilde{H}_{\mathbf{y}_{j}}}} \\
& =\frac{E\left(\tilde{H}_{\mathbf{x}_{i}}-\mu_{\tilde{H}_{\mathbf{x}_{i}}}\right)\left(\tilde{H}_{\mathbf{y}_{i}}-\mu_{\tilde{H}_{\mathbf{y}_{j}}}\right)}{\sigma_{\tilde{H}_{\mathbf{x}_{i}}} \sigma_{\tilde{H}_{\mathbf{y}_{j}}}}
\end{aligned}
$$

where $\operatorname{cov}(.,$.$) is the covariance, \mu_{\tilde{H}_{\mathbf{x}_{i}}}$ and $\mu_{\tilde{H}_{\mathbf{y}_{j}}}$ are the means of $\tilde{H}_{\mathbf{x}_{i}}$ and $\tilde{H}_{\mathbf{y}_{j}}, \sigma_{\tilde{H}_{\mathbf{x}_{i}}}$ and $\sigma_{\tilde{H}_{\mathbf{y}_{j}}}$ are the standard deviations of $\tilde{H}_{\mathbf{x}_{i}}$ and $\tilde{H}_{\mathbf{y}_{j}}$, respectively. $E($.$) is the expec-$ tation of the expression. In this way, the correspondence of $\mathbf{x}_{i}$ in $\mathbf{Y}$ can be found by solving the following optimization problem

$$
\mathbf{y}^{*}=\arg \max _{j}\left\|C\left(\mathbf{x}_{i}, \mathbf{y}_{j}\right)\right\|
$$

\subsection{Registration of two 3D Models}

Once the point-wise correspondence is produced, they can be used for transformation model estimation. Note that due to the multi-modal nature of the spectral bands, influence of noise, and the view changes, keypoints extracted from images may not allow exact point-wise matching. Therefore, the scale of reconstructed 3D models may be different, which makes $3 \mathrm{D}$ registration inaccurate. As a consequence, we need a model that can estimate not only rotation and translation, but also the scale differences between two models.

To solve this problem in an efficient way, we adopted the classic closed form solution based on unit quaternions [8]. This method aims at solving a rigid transformation problem by estimating the rotation, and then recover the scale and translation. The objective of this method is to find a solution to the following minimization problem:

$$
\min \sum_{1}^{n}\left\|R\left(\mathbf{y}_{i}\right)+T-\mathbf{x}_{i}\right\|
$$

where $\mathbf{x}_{i}$ and $\mathbf{y}_{i}$ are $3 \mathrm{D}$ points in two point sets $\mathbf{X}$ and $\mathbf{Y}$ with the same cardinality. $R$ and $T$ are rotation and translation operations, respectively.

The unit quaternion model gives the estimation of one set of matched points. To cope with the uncertainty from the point correspondence step, we adopted the RANSAC algorithm [5] to give a robust estimation of the 3D registration model. The input to the RANSAC are all point-wise correspondences. Minimal sample sets (MSSs) are randomly selected from the input and the model parameters are computed using only the elements of the MSS. Then scaling factor, rotation matrix, and translation vector are calculated based on the MSSs using the unit quaternion model. In the second step, RANSAC checks which elements of the entire dataset are consistent with the model instantiated with the parameters estimated. This iteration continues until the number of matched points are higher than a predefined threshold, or the maximum number of iterations is reached.

\subsection{Registering 3D Hyperspectral Models}

As mentioned previously, the goal of our work is to register band-wise 3D models to produce the complete 3D hyperspectral model following Equation (1). A natural solution is to adopt a progressive strategy so that each $3 \mathrm{D}$ model is only matched to the model from the neighboring band. Let the transformation between two neighboring 3D models $\mathbf{X}(i)$ and $\mathbf{X}(i+1)$ be $\mathcal{T}_{i, i+1}$, suppose the anchor band is $K$, the accumulated transformation for $\mathbf{X}(\lambda)$ is

$$
\mathcal{T}_{\lambda}^{K}=\prod_{i=\lambda}^{K} \mathcal{T}_{i, i+1}
$$

The risk of such an accumulative strategy is that error in $3 \mathrm{D}$ registration may be propagated towards the model built from bands that are far from the anchor band, which will generate noisy final registration results. This solution can not be guaranteed to be optimal because the models from neighboring bands may not be the most compatible. Therefore, it is necessary to find the optimal merge sequence given a set of band-wise 3D models.

To this end, we treat each 3D model as a node in a graph so that each pair of nodes are connected by an edge. The costs associated with the edges are the mean distance errors between the models. The mean distance error among two registered models is calculated as

$$
\mathcal{E}_{\mathbf{X}_{i}, \mathbf{X}_{j}}=\frac{\sum_{i, j} \sqrt{\left(x_{j}-x_{i}\right)^{2}+\left(y_{j}-y_{i}\right)^{2}+\left(z_{j}-z_{i}\right)^{2}}}{n}
$$

where points $\left(x_{i}, y_{i}, z_{i}\right)$ and $\left(x_{j}, y_{j}, z_{j}\right)$ are $3 \mathrm{D}$ points in model $\mathbf{X}_{i}$ and $\mathbf{X}_{j}$ respectively. $n$ is the total number of correspondences between points in two models.

Then we try to find the path with the minimum cost among the graph nodes so that all nodes are visited similar to traveling salesman problem. We adopted a divide and conquer strategy by finding pairs of nodes with the minimum distance and merging each pair to get a new model. The the graph is then updated and this process is repeated until a single registered model is generated.

From implementation point of view, for $n$ models, an $n \times$ $n$ mean error matrix $M$ can be constructed. Note that this matrix may not be symmetric because the error $\mathcal{E}_{\mathbf{X}_{i}, \mathbf{X}_{j}}$ is not necessarily equal to $\mathcal{E}_{\mathbf{X}_{j}, \mathbf{X}_{i}}$ as the transformation matrix may be different. Ignoring main diagonal entries, the lowest 


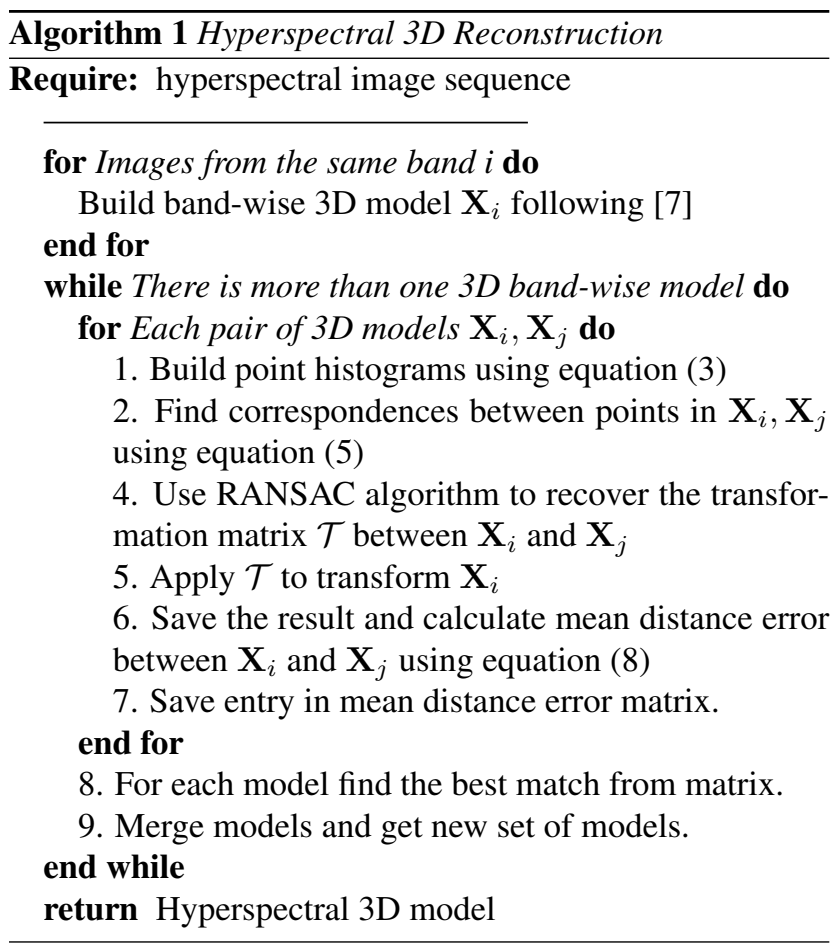

cost associated with merging each model with the other can be calculated by

$$
N_{i}=\min _{j}\left(M_{i, j}\right)
$$

A summary of the hyperspectral 3D reconstruction method is given in the algorithm 1 .

\section{Experiments}

In this section, we first demonstrate the band-wise and the complete 3D models built from hyperspectral data. Then we compare the proposed 3D registration method with several state-of-the-arts methods.

\subsection{D Hyperspectral Model}

The goal of this experiment is to show the differences between the 3D models generated from different hyperspectral

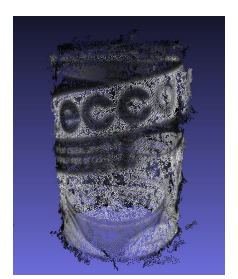

(a)

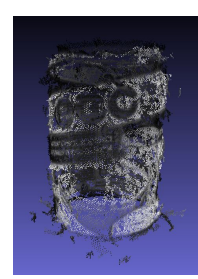

(b)

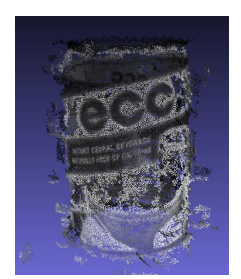

(c)
Figure 3. 3D models generated from (a) 600nm, (b) 700nm and (c) $800 \mathrm{~nm}$ respectively. bands and the difference between the 3D models built from hyperspectral images and RGB images.

We start from showing the results generated on coffee can using method in equation (7). In the experiments, band images from $400 \mathrm{~nm}$ to $560 \mathrm{~nm}$ range and at $1000 \mathrm{~nm}$ were removed because of very low signal to noise ratio. During the 3D reconstruction process, images captured from $880 \mathrm{~nm}$ to $980 \mathrm{~nm}$ have failed to generate complete bandwise 3D models because insufficient number of keypoints can be extracted from some angles. Therefore, in the final reconstruction experiments, we have removed these bands from consideration. This leaves us with 15 bands for bandwise 3D reconstruction and final complete hyperspectral 3D modelling. Fig. 3 shows examples of 3D dense model of the coffee can which are recovered from the point cloud. These 3D models were generated from different hyperspectral bands across the visible to the near-infrared range. It can be seen that each band has captured different structure information of the object. There are substantial overlap on the point sets, but many regions demonstrate complementary information. The complete hyperspectral 3D model combining band-wise 3D models are displayed in Fig. 4(a)(c) from different viewing angles.

To compare the 3D model reconstructed from hyperspectral and RGB images, we also show the $3 \mathrm{D}$ model reconstructed from RGB images in Fig. 4(d)-(f) in the same viewing angle. The results show that the hyperspectral models contain much denser point cloud than the RGB models although the RGB images have higher spatial resolution than the hyperspectral images. The hyperspectral 3D model contains many regions that the RGB models have missed to find any points. These results show the advantage of building $3 \mathrm{D}$

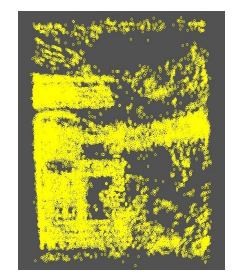

(a)

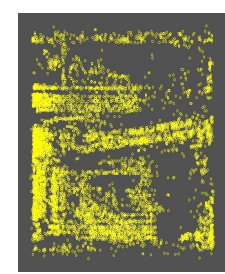

(d)

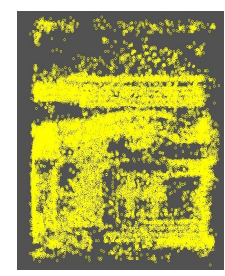

(b)

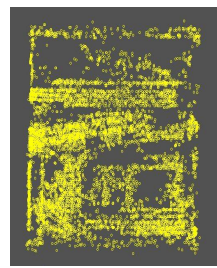

(e)

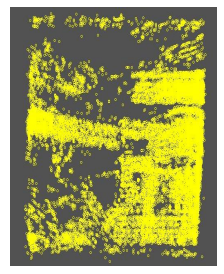

(c)

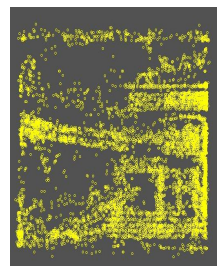

(f)
Figure 4. Comparison of 3D models generated from hyperspectral and RGB images. (a)-(c) 3D models generated from hyperspectral images captured from different viewing angles. (d)-(f) 3D models generated from RGB images at same poses as (a)-(c). 


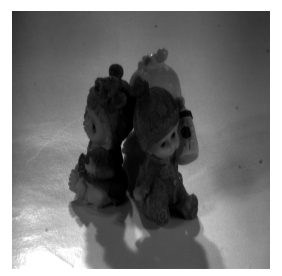

(a)

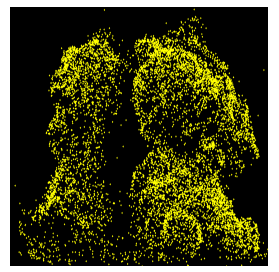

(f)

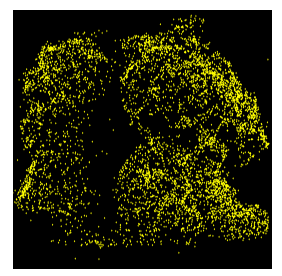

(b)

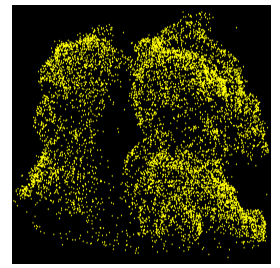

(g)

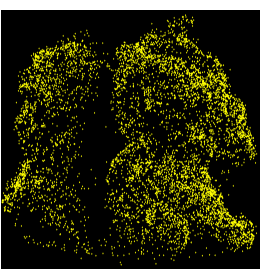

(c)

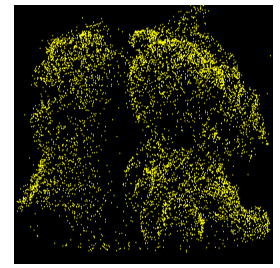

(h)

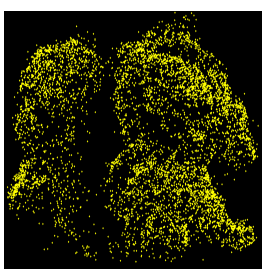

(d)

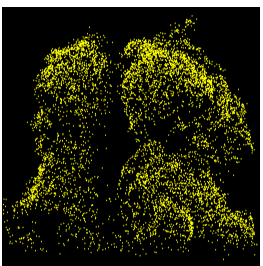

(i)

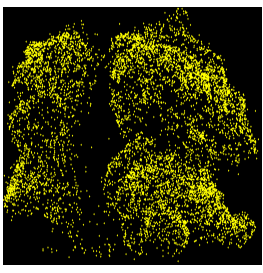

(e)

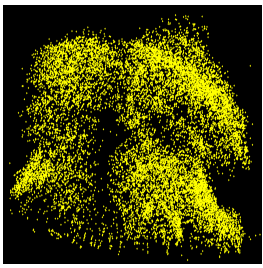

(j)

Figure 5. 3D models generated from hyperspectral images of dolls. (a) Dolls image at 700nm; (b)-(i) 3D models generated from hyperspectral images; (j) Complete hyperspectral 3D model

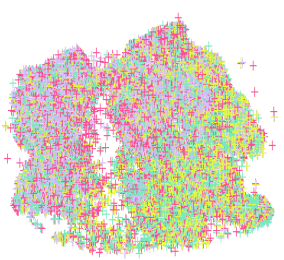

(a)

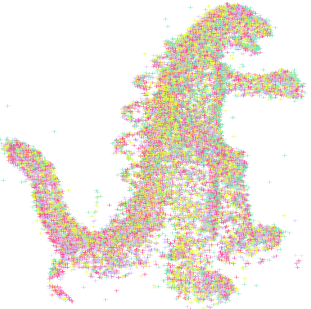

(b)
Figure 6. Band registration of (a) Dolls using 640nm, $660 \mathrm{~nm}, 700 \mathrm{~nm}$ and $720 \mathrm{~nm}$ spectral models ; and (b) dinosaur using red, green, blue, and grayscale models.

models from hyperspectral images, which allows finer and more complete structure information of objects being identified and presented.

Fig. 5 shows eight models of dolls generated from $600 \mathrm{~nm}$ to $740 \mathrm{~nm}$ with $20 \mathrm{~nm}$ in spectral resolution. Images obtained in this range have less noise and the 3D models reconstructed are in better quality. Each of the eight models have fine differences in structure from others. Complete model obtained after registering these eight models is shown in Fig. 5(j). Denser point cloud is generated with more structural details than band-wise models.

We also applied our method on fewer spectral bands of dolls image and RGB models obtained from dinosaur images $^{1}$ so as to present the fine structural details contributed by each models. Fig. 6(a) shows the registration result from four band-wise models with each model represented by different colors. We can see from the figure that each band

\footnotetext{
${ }^{1}$ The dataset is available at http://www.robots.ox.ac.uk/ $\sim$ vgg/data/data-mview.html
}

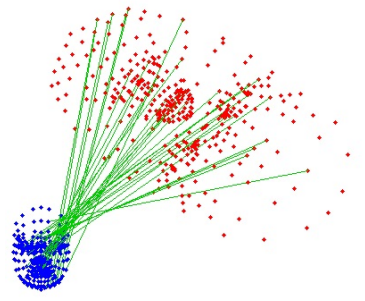

(a)

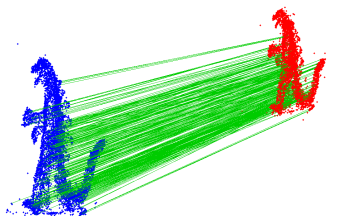

(b)
Figure 7. Sample point correspondences on (a) face models; and (b) dinosaur models.

has contributed to different but complementary structural details. Fig. 6(b) shows the result of registering four different models of dinosaur that are reconstructed from red, green, blue channels and from grayscale images converted from color images. Note that each model has different number of points, scales and rotations.

\subsection{Comparison of 3D Registration Methods}

It is difficult to generate the ground truth of the 3D models built from hyperspectral or RGB images. To give a quantitative comparison of the proposed method and alternative in 3D model registration, we performed experiments on two synthetic dataset. The first dataset is the face point set used in [10]. This dataset contains a sparse 3D face model with 392 points. We then transformed this 3D model using a randomly generated transformation matrix, with scale, rotation, and translation changed. This dataset gives an example of exact point-to-point match. The original and transformed models are shown in Fig. 8(a).

The second dataset is a dinosaur object that has been widely used for 3D modeling ${ }^{1}$. This dataset contains 36 frames of a toy dinosaur captured from different angles. 


\begin{tabular}{|c|c|c|c|c|c|}
\hline & EM-GRBF [10] & RPM [4] & TPS-L2 [10] & CPD [17] & Proposed Method \\
\hline Face & 1.4721 & 1.5016 & 1.6218 & 1.6196 & 0.0014 \\
\hline Dinosaur & 0.3064 & 0.2610 & 0.4318 & 0.2338 & $2.7 \times 10^{-8}$ \\
\hline
\end{tabular}

Table 1. Comparison of accuracy using mean distance error.

\begin{tabular}{|c|c|c|c|c|c|}
\hline & EM-GRBF [10] & RPM [4] & TPS-L2 [10] & CPD [17] & Proposed Method \\
\hline Face & 2.863 & 2.126 & $\mathbf{1 . 5 4 9}$ & 3.360 & 2.706 \\
\hline Dino & 205.532 & 455.157 & 362.344 & 134.683 & $\mathbf{1 3 . 7 7 3}$ \\
\hline Coffee & 210.007 & 310.531 & 313.419 & 90.256 & $\mathbf{1 2 . 6 7 5}$ \\
\hline
\end{tabular}

Table 2. Comparison of efficiency (in seconds).

On the dinosaur model, we generated two 3D models using the red and blue channels, respectively, following the structure from motion approach. This lead to two point sets with 6310 and 7526 points, respectively. This is an example in which some points can not find correspondences. These two models are shown in Fig. $8(\mathrm{~g})$.

We first demonstrate the effectiveness of our point-wise correspondence method. Fig. 7 shows the matched points in the two 3D models on face and dinosaur, respectively. It can be seen that all point correspondences have been detected on the face models, which is relatively simple due to the exact ground truth point-to-point match. The performance of our method on the dinosaur is also excellent, with very few wrong matches been generated. Such results have validated the effectiveness of the point histogram descriptor, which provides statistical information on the structure of objects. Such statistical information is very robust to noises, and more importantly, is invariant to scale changes.

To show the advantages of the $3 \mathrm{D}$ registration method proposed in this paper, we compared our method with EMGRBF [10], RPM [4], TPS-L2 [10], and CPD [17] on both the face and dinosaur datasets. We first compare the accuracy of the registration, whose results are given in Table 1 . It can be seen that our method has significantly outperformed the alternatives with very small mean distance error generated. When efficiency is concerned, Table 2 lists the time to perform the matching tasks by each method. Our method also achieved excellent performance, especially on the dinosaur dataset which has high number of points in both models. Our method does not require iterative point correspondence detection. When combined with quaternion based method which gives a closed-form registration solution, great boosting on the efficiency can be achieved. We also show the registration results in Fig. 8, which give qualitative evaluation of our method and the alternatives.

\section{Conclusions}

We have presented a 3D reconstruction method using hyperspectral images. This method first builds band-wise 3D models. Then a point histogram descriptor is used to find the correspondences between 3D point sets. These pointwise matches are used to register two 3D models and estimate the transformation matrix to recover the scale, rotation and translation parameters. Experimental results on building 3D models from hyperspectral images and RGB images show the benefit of acquiring spectral information for the 3D reconstruction. Our method can capture more detailed structure information of objects being reconstructed. We also performed experiments on synthetic data, which verify the effectiveness and efficiency of the proposed point descriptor and model fusion method. In the future, we will explore other techniques to develop better 3D model which can capture spectral responses more effectively.

\section{References}

[1] S. Belongie, J. Malik, and J. Puzicha. Shape matching and object recognition using shape contexts. IEEE Transactions on Pattern Analysis and Machine Intelligence, 24(24):509522, 2002.

[2] P. Besl and N. McKay. A method for registration of 3-D shapes. IEEE Transactions on Pattern Analysis and Machine Intelligence, 14(2):239-256, 1992.

[3] N. Brusco, S. Capeleto, M. Fedel, A. Paviotti, L. Poletto, G. Cortelazzo, and G. Tondello. A system for 3D modeling frescoed historical buildings with multispectral texture information. Machine Vision and Applications, 17(6):373-393, 2006.

[4] H. Chui and A. Rangarajan. A new algorithm for non-rigid point matching. In Proceedings of the IEEE Conference on Computer Vision and Pattern Recognition, pages 44-51, 2000.

[5] M. Fischler and R. Bolles. Random sample consensus: A paradigm for model fitting with applications to image analysis and automated cartography. Communications of the ACM, 24(6):381-395, 1981.

[6] R. Furbank and M. Tester. Phenomics technologies to relieve the phenotyping bottleneck. Trends in Plant Science, 16(12):635 - 644, 2011.

[7] R. Hartley and A. Zisserman. Multiple View Geometry in Computer Vision. Cambridge University Press, ISBN: 0521540518, second edition, 2004. 


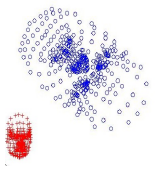

(a)

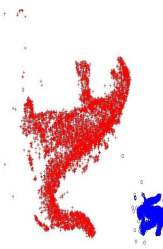

(g)

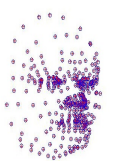

(b)

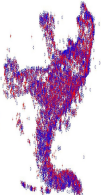

(h)

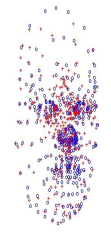

(c)

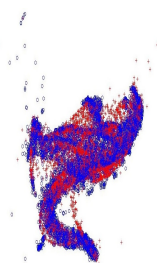

(i)

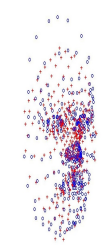

(d)

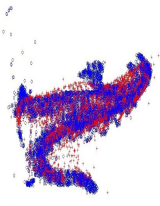

(j)

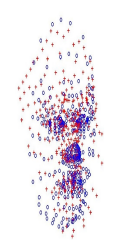

(e)

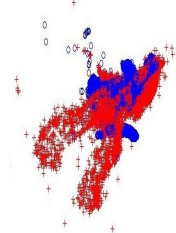

(k)

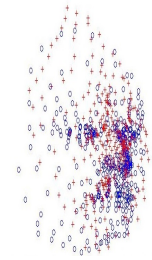

(f)

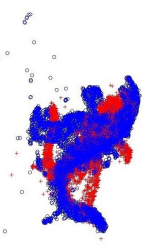

(1)

Figure 8. Comparison of 3D registration results on face and dinosaur data. First row: (a) two 3D face models. Registration results from (b) proposed method, (c) EM-GRBF [10], (d) RPM [4], (e) TPS-L2 [10], (f) CPD [17]. Second row: (g) two 3D dinosaur models. Registration results from (h) proposed method, (i) EM-GRBF, (j) RPM, (k) TPS-L2, (l) CPD.

[8] B. Horn. Closed-form solution of absolute orientation using unit quaternions. Journal of the Optical Society of America A, 4:629-642, 1987.

[9] X. O. J. Garcia, J. Mara Snchez and X. Binefa. Chromatic aberration and depth extraction. In Proceedings of International Conference on Pattern Recognition, pages 1762-1765, 2000.

[10] B. Jian and B. Vemuri. Robust point set registration using Gaussian mixture models. IEEE Transactions on Pattern Analysis and Machine Intelligence, 33(8):1633-1645, 2011.

[11] R. Kawakami, Y. Matsushita, J. Wright, M. Ben-Ezra, Y.-W. Tai, and K. Ikeuchi. High-resolution hyperspectral imaging via matrix factorization. In Proceedings of the IEEE Conference on Computer Vision and Pattern Recognition, pages 2329-2336, 2011.

[12] M. H. Kim, T. A. Harvey, D. S. Kittle, H. Rushmeier, J. Dorsey, R. O. Prum, and D. J. Brady. 3D imaging spectroscopy for measuring hyperspectral patterns on solid objects. ACM Transactions on Graphics, 4(31):38, 2012.

[13] J. Liang, A. Zia, J. Zhou, and X. Sirult. 3D plant modelling via hyperspectral imaging. In Proceedings of the International Workshop on Computer Vision for Accelerated at Bioscience at the International Conference on Computer Vision, 2013.

[14] L. Ma and J. Zhu. Efficient scaling registration algorithm for partially overlapping point set. IET Electronics Letters, 20(49):1267-1268, 2013.

[15] A. Makadia, A. P. IV, and K. Daniilidis. Fully automatic registration of 3D point clouds. In Proceedings of the IEEE Conference on Computer Vision and Pattern Recognition, pages 1297-1304, 2006.

[16] Y. Manabe, S. Kurosaka, and K. Chihara. Simultaneous measurement of spectral distribution and shape. In Pattern Recognition, 2000. Proceedings. 15th International Conference on, volume 3, pages 803-806, 2000.
[17] A. Myronenko and X. Song. Point-set registration: Coherent point drift. IEEE Transactions on Pattern Analysis and Machine Intelligence, 32(12):2262-2275, 2010.

[18] J. Nieto, S. Monteiro, and D. Viejo. 3D geological modelling using laser and hyperspectral data. In 2010 IEEE International Geoscience and Remote Sensing Symposium, pages 4568-4571, 2010.

[19] A. Radosevich, M. Bouchard, S. Burgess, B. Chen, and E. Hillman. Hyper-spectral in-vivo two-photon microscopy of intrinsic fluorophores. Optics Letters, 33(18):2164-2166, 2008.

[20] R. Rusu, N. Blodow, and M. Beetz. Fast point feature histograms (FPFH) for 3D registration. In Proceedings of the IEEE International Conference on Robotics and Automation, pages 3212 - 3217, 2009.

[21] H. N. T. Zinßer, J. Schmidt. Point set registration with integrated scale estimation. In Proceedings of the International Conference on Pattern Recognition and Information Processing, pages 116-119, 2005. 